\title{
Custom-Fit and Lightweight Optimization Design of Exoskeletons Using Parametric Conformal Lattice
}

\author{
Fuyuan Liu ${ }^{1}$, Min Chen ${ }^{1(\bowtie)}{ }^{\left(\text {Lizhe } \text { Wang }^{1}, \text { Xiang Wang }\right.}{ }^{1}$, and Cheng-Hung Lo $^{2}$ \\ ${ }^{1}$ School of Advanced Technology, Xi'an Jiaotong - Liverpool University, Suzhou, China \\ min. chenexjtlu.edu.cn \\ 2 School of Film and TV Arts, Xi'an Jiaotong - Liverpool University, Suzhou, China
}

\begin{abstract}
This paper presents an integrated design method for the customization and lightweight design of free-shaped wearable devices, illustrated by a lower limb exoskeleton. The customized design space is derived from the 3D scanning models. Based on the finite element analysis, the structural framework is determined through topology optimization with allowable strength. By means of generative design, the lattice library is constructed to fill the frames under different conformal algorithms. Finally, the proposed method is illustrated by the exoskeleton design case.
\end{abstract}

Keywords: Custom-fit · Lightweight design $\cdot$ Conformal lattice $\cdot$ Generative design

\section{Introduction}

Civilian wearable robots, specialized as exoskeletons, are in vigorous development due to the increasing demands from mobility defection and the rehabilitation of aged people [1]. Enhanced strength and endurance are the functional expectations of those devices, while conformability and aesthetic property are the critical aspects considered by the users [2]. To satisfy functional expectations and user requirements, there are several challenges required to solve, involving lightweight optimization and adaptation of individual differences [3].

The reduced mass leads to less resistance to the motion and also less harm to human beings in the case of collision [4]. For the lightweight of wearable structures, typically there are two solutions: to choose lighter material but sufficient strength or to optimize the structure design [5]. Currently, aluminium alloy is applied in the most available exoskeleton products. One of the key reasons is due to its good balance between mass and cost [6]. For lightweight material, carbon fibre composites and titanium, both with extremely high strength and low density, are occasionally adopted in some special cases 
[7]. The application of advanced material is not a good choice due to the high expense. Therefore, using topology optimization to reduce the structure mass is a typical way for lightweight design. However, most cases are for solid material [8]. With the development of additive manufacturing, there is one more possibility to minimalize the mass by using lattice material, which is designed for higher specific strength (the ratio between strength and density) [9]. Although many optimization schemes using predesigned lattice structures have been proposed, it is yet to practice on the lightweight design of exoskeletons.

Besides lightweight, customization is another crucial factor for wearable devices. Custom-fit here indicates the fitting of physical shape and the customer's preferred pattern. Different users have different physiological conditions, like height, weight and gait pattern. The prescribed size, form and trajectories of exoskeletons are required to match individuals for a snug fit [10]. Using 3D scanning technology is an effective way to get the user-specified digital model [11], which provided an accurate reference for customizing exoskeletons. However, previously it is not feasible to provide various appearances due to the limitation of manufacturing and the design approaches. Generative design is a method that is capable of creating unrepeatable forms under certain principles and mathematics algorithms [12,13], which can develop aesthetic and functional performance.

In this paper, conformal lattices, based on the generative design method, is proposed to enhance the lightweight and custom-fit purpose. The cellular structure can be expanded in a novel way through specific rules.

\section{Multi-dimensional Customized Design Method}

To address the Lightweight and Custom-fit challenges, an integrated approach is proposed here to cope with the design of the exoskeleton. The overall strategy is illustrated in Fig. 1, which mainly contains three parts:

1. Tailoring free shape modelling based on 3D scanning and reverse engineering

2. Refining the robust design frame with topology optimization

3. Mapping conformal lattice 


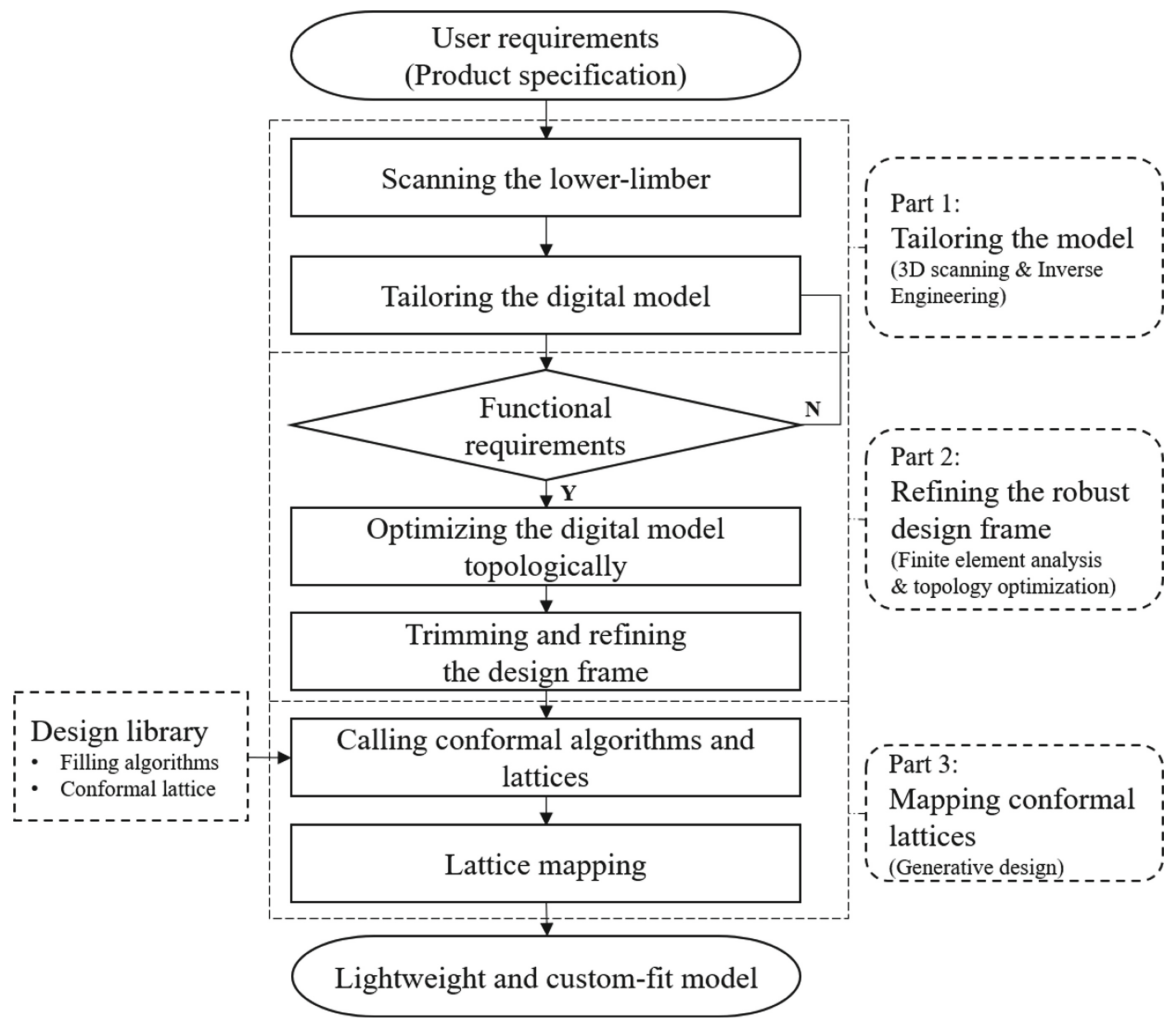

Fig. 1. Flowchart of the integrated design method

\subsection{Design Domain and Modelling}

For the custom-fit design purpose, the 3D scanning was used to obtain the digital customer models, from which the closely fit design space was derived, as illustrated in Fig. 2. Here a uniform thickness of $5 \mathrm{~mm}$ is assumed for the aluminum plate. The material properties are shown in Table 1.

Table 1. Material properties of aluminum

\begin{tabular}{l|l|l|l}
\hline Young's Modulus $(\mathrm{GPa})$ & Poisson's ratio & Yield strength $(\mathrm{MPa})$ & Density $\left(\mathrm{kg} / \mathrm{m}^{3}\right)$ \\
\hline 76.6 & 0.33 & 250 & 2670 \\
\hline
\end{tabular}

\subsection{Numerical Analysis Based on Topology Optimization}

To achieve an optimized morphology with specific boundary condition and constraints for the design space of a thigh, finite element analysis is executed in this stage. Load 


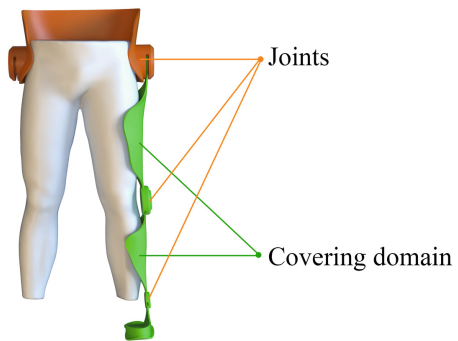

Fig. 2. Custom-fit initial design domain

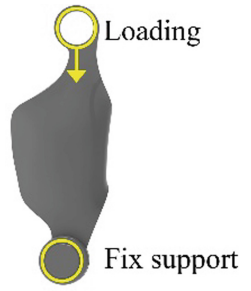

Fig. 3. Loading and boundary conditions

condition is regarded as when the upper body is supported entirely by the exoskeleton in the static mechanical model, proving the stability of the thigh shell structure. According to GB/T 10000-1988, the allowable force formula is expressed in (1):

$$
F_{\text {max }}=m g \times n_{h} \times n_{s}
$$

where, the weight of consumer: $m$; safety factor: $n_{s}=1.5 ; g=10 \mathrm{~m} / \mathrm{s}^{2}$; the ratio of upper body mass: $n_{h}=65.6 \%$

According to the formula, the joint is set as fix support and $800 \mathrm{~N}$ force acting on the joint of the thigh exoskeleton, as shown in Fig. 3. After a specific load condition, meshing sensitivity analyses are carried out to obtain a reasonable element mesh size for an effective simulation at a low computational cost.

In Table 2, the sensitivity analysis of mesh density is conducted. Max stress stabilizes gradually and arrives at a peak when mesh size is set as $3 \mathrm{~mm}$; and the number of mesh is increasing dramatically as decreasing mesh size, especially appearing a sharp increase from $3 \mathrm{~mm}$ to $2.5 \mathrm{~mm}$. It means that the mesh size could be set as $3 \mathrm{~mm}$ for a considerable result in an affordable time.

Table 2. Mesh sensitivity analysis

\begin{tabular}{l|l|l|l|l}
\hline Max element size (mm) & 5 & 4 & 3 & 2.5 \\
\hline Nr. Elements & 33967 & 73099 & 154010 & 428755 \\
\hline Max Stress (MPa) & 206.6 & 202.77 & 212.31 & 211.44 \\
\hline
\end{tabular}

The conventional topology optimization method, namely solid isotropic material with a penalty (SIMP), was used for initial optimization in this study. SIMP was an element density-based optimization method by making a density approach to a specific range.

This element density, namely pseudo density as design variables, was used for topology optimization. This pseudo density value was set in the range of 0 to 1 , presenting the void and solid element. The value between 0 to 1 was used to prevent the stiffness matrix from scaling linearly during the solution process. Therefore, with the stiffness at 
each iteration, the interpolation function was presented by the expression of power law (2):

$$
P\left(\rho_{i}\right)=\rho_{i}^{P}
$$

where $\rho_{i}$ is pseudo-density variables and $P$ is penalty factor (stiffness), $P$ is set to be 3 in this study.

The objective function for each element is to find $\rho_{i}$ satisfying the minimum overall compliance of structure (3):

$$
\text { min: overall compliance of structure }=\frac{1}{2} F^{T} U
$$

where, $\mathrm{U}$ and $\mathrm{F}$ present the nodal displacement vector and the external load vector, respectively.

Meanwhile, the volume and the static equilibrium should be satisfied (4):

$$
\begin{gathered}
\sum_{i=1}^{n} \rho_{i} v_{i}=V \leq V_{\text {upper bound }} 0<\rho_{i} \leq 1, i=1,2,3, \ldots, n \\
F=K U
\end{gathered}
$$

where, $\mathrm{V}$ is the initial volume of the target domain with upper bound, $v_{i}$ is the volume of the i-th element.

The objective function here is the minimal mass, which is equivalent to minimal volume with the same density condition, as shown in Table 3.

Table 3. Original domain and its optimizations

\begin{tabular}{c|c|c|c}
\hline Original domain & Opt.1(50\%) & Opt.2(30\%) & Smoothed model(51\%) \\
\hline & & & \\
& & & \\
\hline
\end{tabular}

However, those topology results are fragmentized and irregular structure far from an available product, which needs further design and modifies. Hence, setting the lightweight optimization as the functional target, the shape is trimmed according to material distribution, as shown in Fig. 4. To ensure that the lightweight result follows the scientific and accurate analysis, the strength of the result trimmed is analysed, and the maximum stress is $207.23 \mathrm{MPa}$, less than the yield strength of Aluminium. This provides an effective design frame for the lightweight optimization with lattice structure. 


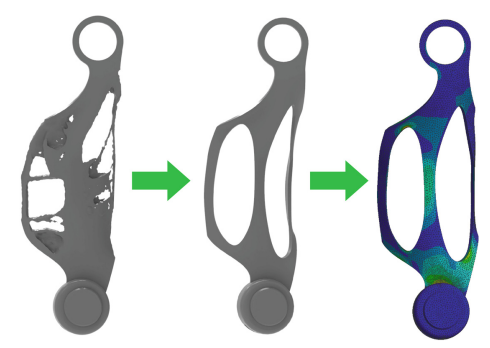

Fig. 4. Topology optimization and remodeling

\subsection{Conformal Lattice Generative Design}

Filling conformal lattice works for 2.5D non-concave design domain, which is constructed by trimming or extruding; mainly two conditions are considered, the boundary conformal condition and the surface conformal condition, illustrated in Fig. 5.

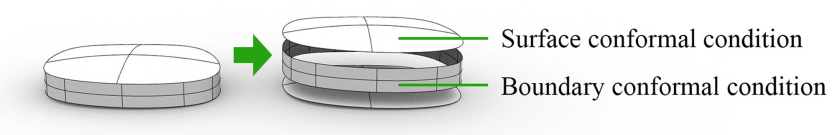

Fig. 5. Conformal conditions

Four proposed algorithms of filling conformal lattice are described in response to the loading condition, as shown in Table 4.

a. Voxel-like conformal filling.

A series of voxel-like cells are populated on the non-concave design domain, which can be filled with tailored lattices. This algorithm covers four parts including adjusting UV direction, setting a unit cell, tessellating, trimming. In response to the loading, UV direction is manipulated and adjusted by four control points $a_{o}, b_{o}, c_{o}, d_{o}$ which are located on the boundary of a NURBS surface derived from non-concave surface. Following that, the unit cell is represented by $S_{O^{-}}$the length of lattice and $T_{o^{-}}$the thickness of lattice; the NURBS surface is tessellated to voxel-like cells. In order to trim cells out of the design domain, each voxel cell is assigned a logic value (i.e., one or zero), with one denoting a filling space and zero indicating void space.

Assuming the trimming result is denoted by design domain $\mathrm{E}$, unit cell $\mathrm{F}$ and the filling cells are G, this can be represented by (5).

$$
G_{n}=\left\{\begin{array}{l}
1, \text { if }(E) \cap\left(f\left(F_{n}\right)\right) \\
0, \text { otherwise }
\end{array}\right.
$$

where $n$ is each cell indices, $f\left(F_{n}\right)$ is an operation on $F_{n}$ to determine the location of each cell. Grasshopper's "Morph to Twisted Box" component can be used to perform lattice filling function. 
Table 4. Four conformal algorithms

\begin{tabular}{|c|c|}
\hline $\begin{array}{ll}1 \\
1\end{array}$ & $\begin{array}{l}\text { a. Voxel-like conformal filling } \\
T_{o} \text { - the thickness of the lattice } \\
S_{o} \text { - the length of the lattice } \\
a_{o}, b_{o}, c_{o}, d_{o} \text { - control points of } \mathrm{UV} \text { direction } \\
k_{h} \text { - fractal parameter }\end{array}$ \\
\hline & $\begin{array}{l}\text { b. Boundary shrinkage conformal filling } \\
T_{o} \text { - the thickness of the lattice } \\
k_{b} \text { - the hoop gradient } \\
k_{s} \text { - the radial gradient } \\
k_{h} \text { - fractal parameter }\end{array}$ \\
\hline & $\begin{array}{l}\text { c. Voronoi conformal filling } \\
T_{o} \text { - the thickness of the lattice } \\
k_{b} \text { - the hoop gradient } \\
k_{s} \text { - the radial gradient }\end{array}$ \\
\hline & $\begin{array}{l}\text { d. Triangular mesh conformal filling } \\
T_{o} \text { - the thickness of the lattice } \\
k_{b} \text { - the hoop gradient } \\
k_{p} \text { - the scattering parameter }\end{array}$ \\
\hline
\end{tabular}

Additionally, for obtaining optimal lattice structure, a fractal filling algorithm is constructed for various applications.

Assuming the fractal result is denoted by fractal parameters $k_{h}$, fractal domain D, fractal cell $\mathrm{R}$, this can be represented by (6).

$$
\mathrm{T}=f\left(R_{m}, k_{h}\right) \quad R_{m}=\left\{\begin{array}{l}
1, \text { if }(D) \cap\left(f\left(G_{m}\right)\right) \\
0, \text { otherwise }
\end{array}\right.
$$

where $m$ is each cell indices, $f\left(G_{m}\right)$ is an operation on $G_{m}$ to determine the location of each cell, $f\left(R_{m}, k_{h}\right)$ is an operation on $R_{m}, k_{h}$ to determine the fractal quantity of each cell. Grasshopper's "Subdivided Twisted Box" component can be used to perform the fractal function.

This voxel-like filling algorithm remains the structure feature of each lattice; the population direction of the lattice can be adjusted in response to the loading. The disadvantage of this algorithm is that the lattice structure has a 'zigzag' boundary, which may result in the stress concentration. 
b. Boundary shrinkage conformal filling.

For this algorithm, the contour profile of the filling domain is shrinking in the inward direction to the centroid. The population of the lattices can be determined by $k_{b}$, defined as the hoop gradient, as well as $k_{s}$, expressed as the radial gradient. These two parameters are employed to construct shrinking cells to fill lattice. This method avoids the 'zigzag' boundary but twists filling cells. However, the form of shrinking conformal population results in the dense population along the radial direction and an $\mathrm{N}$-side blank region sited in the centre, which cannot populate the cube lattice in the centre.

c. Voronoi conformal filling.

Analogous to Boundary shrinkage conformal filling, $k_{b}$-the hoop gradient and $k_{s}$-the radial gradient, as decisive parameters, are employed to construct a dot array, by which the Voronoi diagram is built. The diagram is projected on the non-concave surface for thickening to lattice structure. This filling method is aesthetic but natural; however, the machinal properties of lattice are difficult to measure and verify.

d. Triangular mesh conformal filling.

Different from the conformal way of the boundary shrinkage, this algorithm constructs the lattice structure from the centroid to the contour profile through the line frame. $k_{p}$, defined as the scattering parameter, is applied to construct the radio dot array in the outward direction to the circumference.

Assuming the number of the dots in each boundary shrinkage level is denoted by the scattering parameter $k_{p}$ and boundary shrinkage level $\mathrm{B}$, this can be represented by (7).

$$
P_{b}=k_{p} B \quad B \in\left[0, \ldots, k_{b}\right]
$$

where Grasshopper's "Divided Curve” component can be used to construct a dot array.

Delaunay mesh is built based on the radio dot array. Following that, more complex wireframes are constructed and thickened to the lattice structure. This algorithm will lead to better-proportioned mesh. Although organic and aesthetic is lattice structure, the mechanical properties of lattice structure is hard to verify and measure. Besides, this algorithm is just appropriate for non-concave space with small curvature.

Generally, the lattice can be designed and populated in the design domain within the frame of the various algorithms. Based on these four algorithms, the exoskeleton can be optimized with an customized and lightweight form, illustrated in Fig. 6. 


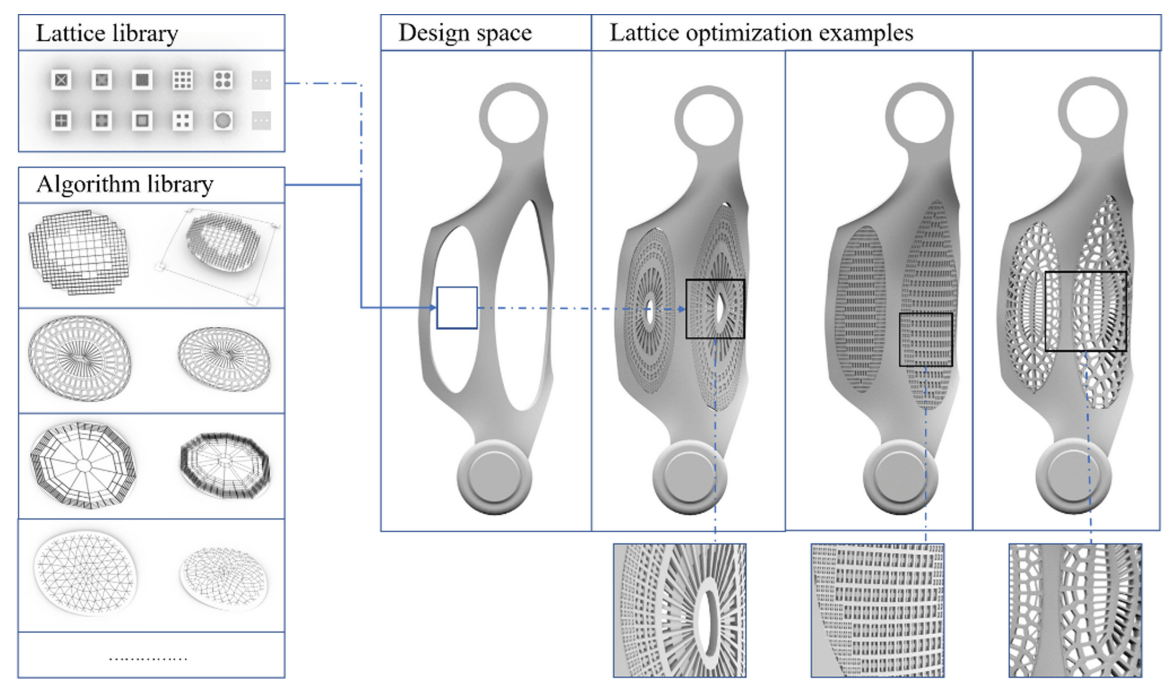

Fig. 6. Customized design using lattice library

\section{Conclusion}

In this paper, an integrated method for the free shape customization and lightweight design is represented thoroughly with the case of the lower limb exoskeleton. The research fruits can be demonstrated as follows:

1) This design method is instrumental in designing customized wearable robots from form to structure. It not only brings a snug fit for different users through 3D scanning but also offers myriad optimal choices via generative conformal lattice, which is able to satisfy flexibly the customized requirements of different individuals.

2) Topology optimization provides a well-defined material distribution basis in order to construct effective 2.5D non-concave design domains in which conformal lattice is filled. The incorporation of both lattice structure and topology optimization is effective to design a functional and lightweight wearable product.

3) This method presents a subtle design process from customization to structural optimization with various advanced technologies, including 3D scanning, topology optimization and generative design, which provides a constructive reference related to customized and lightweight products for the designers in the exploration of AM's design space.

Acknowledgement. The research is financially supported by National Natural Science Foundation of China (51805447), XJTLU Key Program Special Fund (KSF-E-01, KSF-E-27) and Research Development Fund (RDF-17-02-44), Open Project for Vehicle Application Engineering of Beijing Jiaotong University (BMRV20KF03). 


\section{References}

1. Chen, G., Chan, C.K., Guo, Z., Yu, H.: A review of lower extremity assistive robotic exoskeletons in rehabilitation therapy. Crit. Rev. Biomed. Eng. 41(4-5), 343-363 (2013)

2. Yang, C., Zhang, J., Chen, Y., Dong, Y., Zhang, Y.: A review of exoskeleton-type systems and their key technologies. Proc. Inst. Mech. Eng. C J. Mech. Eng. Sci. 222(8), 1599-1612 (2008)

3. Sanchez-Villamañan, M., Gonzalez-Vargas, J., Torricelli, D., Moreno, J., Pons, J.: Compliant lower limb exoskeletons: a comprehensive review on mechanical design principles. J. Neuroeng. Rehabil. 16(1), 55-71 (2019)

4. Li, A., Liu, C.: Lightweight design of a crane frame under stress and stiffness constraints using super-element technique. Adv. Mech. Eng. 9(8), 1-15 (2017)

5. Wang, X., Zhang, D., Zhao, C., Zhang, P., Zhang, Y., Cai, Y.: Optimal design of lightweight serial robots by integrating topology optimization and parametric system optimization. Mech. Mach. Theory 132, 48-65 (2019)

6. Young, A., Ferris, D.: State of the art and future directions for lower limb robotic exoskeletons. IEEE Trans. Neural Syst. Rehabil. Eng. 25(2), 171-182 (2017)

7. Sawicki, G., Ferris, D.: A pneumatically powered knee-ankle-foot orthosis (KAFO) with myoelectric activation and inhibition. J. Neuroeng. Rehabil. 6(1), 23-39 (2009)

8. Wu, S., Tu, J., Li, J., Li, J., Li, X., Wei, S.: Research on modeling and structural optimization design of lower extremity rehabilitation exoskeleton. Mech. Sci. Tech. Aerosp. Eng. 39(6), 891-897 (2020)

9. Nguyen, J., Park, S., Rosen, D., Folgar, L., Williams, J.: Conformal lattice structure design and fabrication. In: 23rd Annual International Solid Freeform Fabrication Symposium - An Additive Manufacturing Conference, SFF 2012, pp. 138-166 (2012)

10. Hill, D., Holloway, C., Morgado-Ramirez, D., Smitham, P., Pappas, Y.: What are user perspectives of exoskeleton technology? A literature review. Int. J. Technol. Assess. Health Care 33(2), 160-167 (2017)

11. Rossi, S., Patanè, F., Del Sette, F., Cappa, P.: WAKE-up: a wearable ankle knee exoskeleton. In: Proceedings of the IEEE RAS and EMBS International Conference on Biomedical Robotics and Biomechatronics, pp. 504-507 (2014)

12. Agkathidis, A.: Generative Design, pp. 16-17. Laurence King Publishing Ltd., London (2015)

13. Nordin, A.: Reconciling Form and Function through Generative Design. Division of Machine Design, Department of Design Sciences, Faculty of Engineering LTH, Lund University, pp. 17-47 (2015)

Open Access This chapter is licensed under the terms of the Creative Commons Attribution 4.0 International License (http://creativecommons.org/licenses/by/4.0/), which permits use, sharing, adaptation, distribution and reproduction in any medium or format, as long as you give appropriate credit to the original author(s) and the source, provide a link to the Creative Commons license and indicate if changes were made.

The images or other third party material in this chapter are included in the chapter's Creative Commons license, unless indicated otherwise in a credit line to the material. If material is not included in the chapter's Creative Commons license and your intended use is not permitted by statutory regulation or exceeds the permitted use, you will need to obtain permission directly from the copyright holder.

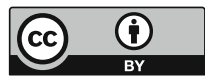

